Itinéraires Itinéraires

Littérature, textes, cultures

2012-3 | 2013

Lire les villes marocaines

\title{
Dionysos à Meknès : les dieux, les corps et la ville chez Miloudi Chaghmoum
}

\section{Ziad Elmarsafy}

\section{OpenEdition}

\section{Journals}

Édition électronique

URL : http://journals.openedition.org/itineraires/994

DOI : 10.4000/itineraires.994

ISSN : 2427-920X

Éditeur

Pléiade

\section{Édition imprimée}

Date de publication : 1 juillet 2013

Pagination : 127-135

ISBN : 978-2-343-01183-7

ISSN : 2100-1340

Référence électronique

Ziad Elmarsafy, «Dionysos à Meknès : les dieux, les corps et la ville chez Miloudi Chaghmoum », Itinéraires [En ligne], 2012-3 | 2013, mis en ligne le 01 décembre 2012, consulté le 20 avril 2019. URL: http://journals.openedition.org/itineraires/994 ; DOI : 10.4000/itineraires.994

\section{cc) (i) (9)}

Itinéraires est mis à disposition selon les termes de la licence Creative Commons Attribution - Pas d'Utilisation Commerciale - Pas de Modification 4.0 International. 


\title{
Dionysos à Meknès : les dieux, les corps et la ville chez Miloudi Chaghmoum*
}

\begin{abstract}
This article explores the intersection of the urban, the theological and the mystical in Miloudi Chaghmoum's novel, Masālik Al-Zaytūn [Routes through the Olive Trees / The Routes of Meknes] through an analysis of the novel's repeated allusions to the idioms of Sufism and the figure of Dionysus. Chaghmoum's retelling of a traumatic narrative - namely that of a migrant to Meknes who winds up committing a crime without realizing it - raises key questions regarding the place of the individual in urban space, which emerges as a threatening environment rather than a place of safety.
\end{abstract}

Keywords : Miloudi Chaghmoum, novel, city, Sufism, space

Mots clés : Miloudi Chaghmoun, roman, cité, soufisme, espace

Dans l'œuvre de Miloudi Chaghmoum ${ }^{1}$, c'est surtout le roman Masālik Al-Zaytoun (Passages des oliviers) qui retiendra mon attention ${ }^{2}$, mais aussi bien pourrait-on traduire ce titre par Passages de Meknès, puisque Meknès est couramment désignée par l'expression Meknès Al-Zaytoun, et que l'olivier reste le symbole et le synonyme de Meknès. L'argument est simple : il s'agit de décrire les moyens utilisés par Chaghmoum pour élucider les rapports entre le sacré et la création littéraire via la figure de

*. Je tiens à remercier Souad Eddouada, Philippe Chevrant et Marc Kober pour leur aide et leur soutien pendant la préparation de cet article.

1. Né en 1947 à Ben Ahmed au Maroc, diplômé et docteur ès philosophie des sciences, Miloudi Chaghmoum enseigne à la faculté de lettres de l'université de Meknès. Auteur de plusieurs romans et de recueils, il a reçu le prix du livre marocain en 2000.

2. Mon analyse trouve son inspiration initiale dans un chapitre consacré à la question du corps par Hicham Al-Alaoui, الجسد والمعن، : قر اعات في، السبرة الر و ائية المغر بية [Le Corps et la signification: lectures du roman marocain], Casablanca, Al-Madāris, 2006, p. 93-125. Je tiens à préciser toutefois qu'il y a plusieurs écarts entre son analyse et la mienne, surtout par rapport à la représentation urbaine et par rapport à la dimension «théologique» de ce roman. 
la ville, qui devient une extension et une allégorie du narrateur. Avant de commencer l'analyse du texte et du rôle joué par la ville dans le roman, il est utile de préciser quelques points au sujet de ce romancier complexe. Son écriture s'étend à l'histoire des sciences - le nom d'Henri Poincaré revient à plusieurs reprises dans ses livres et ses essais - au mysticisme ainsi qu'à la littérature ${ }^{3}$. En 1991, l'année qui suit la publication de Masālik Al-Zaytoun, il a publié une étude importante sur l'imaginaire et le sacré dans le soufisme ${ }^{4}$. Le style de Miloudi Chaghmoum est marqué par une narration très consciente d'elle-même et une réflexion ludique sur le statut du narrateur et l'acte de raconter qui rappelle Borges et Calvino. Il en résulte que beaucoup de lecteurs trouvent ses romans difficiles, accusation à laquelle il répond en revendiquant la responsabilité de l'écrivain : «Je n'écris pas pour ceux qui lisent la littérature pour s'endormir ${ }^{5}$. » Il écrit pour rendre le monde moins familier et éveiller la curiosité du lecteur.

Mes propos sont bâtis sur les thèses de Thomas Pavel sur le roman et celles de Michel de Certeau sur l'espace. Pour Pavel, le roman est le genre de l'individualisme, compris en termes anthropologiques plutôt que dans un cadre libéral. S'appuyant sur les théories de Louis Dumont, pour qui l'individu est à l'origine l'ascète qui échange ses droits sociaux pour se vouer à un dieu tutélaire, lequel le protège et remplace la société comme matrice, Pavel regarde le roman comme le genre qui pose la question axiologique suivante : comment est-il possible pour l'individu d'habiter le monde où il voit le jour? Quel est le rapport - si rapport il y a - entre l'idéal moral qui informe $1^{\prime}$ individu et 1 'ordre de ce monde ${ }^{6}$ ? En ces termes, la théorie de Pavel n'est pas très loin de ce qui pousse Miloudi Chaghmoum lui-même à écrire. Le romancier se demande comment un auteur marocain peut exprimer son humanité 7 .

$\mathrm{Vu}$ l'importance du lieu dans les romans de Chaghmoum, et surtout dans Masālik Al-Zaytoun, il serait utile de prendre en considération l'idée de l'espace et son utilisation dans le discours du récit. Michel de Certeau a vu la narration comme ce qui transforme un lieu donné en un espace : l'espace est, selon cet auteur, un lieu pratiqué. C'est précisément la pratique du texte - la lecture, l'écriture, la récitation - qui métamorphose un lieu en espace :

3. Salim Jay, Dictionnaire des écrivains marocains, Casablanca et Paris, Eddif et ParisMéditerranée, 2005, p. 123-124.

4. Miloudi Chaghmoum, المتخيل و القسي في التصوف الاسلامي: الحكاية و البركة [L'Imaginaire et le sacré dans le soufisme : le récit et la bénédiction], Meknès, Al-Majlis Al-Baladī, 1991.

5. Miloudi Chaghmoum, 《 أنا لا أكتب للناس الذين يقر أون الأدب لينامو ا Je n'écris pas pour ceux qui lisent la littérature pour s'endormir »], Al-Sharq Al-Awsat, 19 décembre 2001, p. 19.

6. Thomas Pavel, La Pensée du roman, Paris, Gallimard, 2003, p. 46-47.

7. Telle est la question posée sur le site de l'auteur: www.chaghmoum.net. 
Ainsi la rue géométriquement définie par un urbanisme est transformée en espace par des marcheurs. De même, la lecture est l'espace produit par la pratique du lieu que constitue un système de signes - un écrit ${ }^{8}$.

De plus, cette pratique a des effets importants sur le plan social et juridique. La légitimité d'un acte dépend du récit qui le précède :

Tel est précisément le rôle premier du récit. Il ouvre un théâtre de légitimité à des actions effectives. Il crée un champ qui autorise des pratiques sociales risquées et contingentes [...]. Avant le jugement régulateur, il y a le récit fondateur ${ }^{9}$.

Le roman a partie liée avec ce processus de légitimation, surtout par le biais des récits qui rendent un lieu ou une action intelligible à travers l'invocation des divinités qui veillent sur eux et les protègent.

Publié en 1990, Masālik Al-Zaytoun pourrait se lire comme un hymne d'amour à la ville de Meknès. Le nœud du récit est une histoire assez simple : un homme quitte son village dans le sud pour s'établir à Meknès et tue une de ses collègues de travail. Au lieu de raconter cette fabula en ordre chronologique, le roman est organisé en suivant la géographie de Meknès. La première partie est intitulée " Masālik al-mā " » (Passages des eaux), ce qui en dit long sur la sensibilité du romancier Chaghmoum, qui comprend la géographie physique de la ville : il commence par les canaux d'irrigation qui soutiennent les oliviers et, partant, la ville de Meknès ${ }^{10}$. Les chapitres suivants portent pour titre les portes de la ville et les épithètes attachées au mot « zaytoun » (olive); ainsi « L'olive rouge », « olives sèches », « olives amères ", « Le plaisir de l'olive ». En même temps, les descriptions de la ville ne sont jamais loin du sacré. La description de l'état de dévastation de la ville est rédigée en empruntant le style du Coran : "Considère comment les chiens ont été réveillés, comment les vallées ont été ouvertes, et comment les montagnes ont été aplanies ${ }^{11}$. » Les états affectifs et psychologiques du narrateur sont également décrits en référence au Coran : « La fatigue se révèle sous forme de versets dont certains sont figuratifs et nul ne connait leur interprétation à part les cyniques et ceux qui sont enracinés

8. Michel de Certeau, L'Invention du quotidien, 1 : Arts de faire, Paris, Gallimard, 1990, p. 173. 9. Ibid., p. 183-185.

10. Sur l'importance de cet aspect de la géographie urbaine de la ville de Meknès, voir Calogero Montalbano, « Dar Al-Ma: The Architecture of Water in the Islamic Countries », dans Salma K. Jayyusi et al. (dir.), The City in the Islamic World, Leiden et Boston, Brill, 2008, p. 711-712.

11. Miloudi Chaghmoum, الأعمال الكاملة [Euvres complètes], Rabat, Dār al-Thaqāfa, 2005, t. I, p. 477. La phrase de Chaghmoum est basée sur les versets 17-20 de la sourate 88 : « Ne considèrent-ils pas / Comment les chameaux ont été créés; / Comment le ciel a été élevé; / Comment les montagnes ont été placées; / Comment la terre a été aplanie? » (Les citations du Coran sont empruntées à l'édition suivante : Le Coran, traduction et notes par Denise Masson, Paris, Gallimard, 1967). 
dans les sciences de la fatigue ${ }^{12}$. " Sous un angle plus large, le texte de Chaghmoum ne fait que reprendre une des premières tâches du roman, au moins dans sa forme moderne (à partir de 1800), à savoir enquêter sur l'ordre social urbain et les forces naturelles et surnaturelles qui risquent de le déstabiliser ${ }^{13}$. Ces forces viennent du monde naturel qui entoure la ville d'une part, mais d'autre part de l'intérieur imprévisible de l'homme urbain et de ses rapports avec les dieux ou avec son dieu. Cette imprévisibilité joue autant sur les aspects aléatoires de la langue que sur le comportement souvent incompréhensible des personnages.

Le roman commence par une invocation liée à la question de l'imprévisible. Le paratexte (Chaghmoum l'intitule Tahdhìr ou « avertissement») cite le livre de Abd al-Wahhāb al-Cha 'rānī, un soufi égyptien du Xvi' siècle et auteur d'une encyclopédie biographique des soufis, Lawaqih al anwār fi tabaqāt al akhyār [Lumières sur les hiérarchies des vertueux] ${ }^{14}$. Le texte cité vient de la préface de l'œuvre, dans laquelle Sha 'arānī propose une apologie du soufisme, et avertit le lecteur du fait qu'il ne faut pas tout interpréter uniquement selon le sens propre des mots, surtout quand il s'agit de poésie, domaine privilégié de l'expression mystique. Il faut distinguer entre le sens apparent (al-zāahir) et le sens caché (al-bātin); le propre et le figuré. Le texte de Cha 'rānī cité dans le roman de Chaghmoum raconte l'anecdote suivante pour illustrer cette problématique et ses conséquences : un cheikh ( 'àlim, un juriste) de Bagdad va à la mosquée. En route il entend un ivrogne qui chante les vers suivants :

Après le 20 du mois de Cha 'bān ${ }^{15}$, continue à boire jour et nuit et ne bois pas dans un petit gobelet, il n'est plus temps pour cela.

12. Miloudi Chaghmoum, Euvres complètes, t. I, op. cit., p. 490. La phrase de Chaghmoum est une allusion au verset 7 de la sourate 3, où il est question des différents aspects et niveaux d'interprétation qu'on trouve dans le Coran : «C'est lui qui a fait descendre sur toi le Livre. / On y trouve des versets clairs / la Mère du Livre - / et d'autres figuratifs. / Ceux dont les cœurs penchent vers l'erreur / s'attachent à ce qui est dit en figures / car ils recherchent la discorde / et ils sont avides d'interprétations; / mais nul autre que Dieu / ne connaît l'interprétation du Livre. / Ceux qui sont enracinés dans la Science disent : / "Nous y croyons! / Tout vient de notre Seigneur !" / Mais seuls, / Les hommes doués d'intelligence s'en souviennent. »

13. Voir l'analyse de cette question dans l'étude de Richard Lehan, The City in Literature: An Intellectual and Cultural History, Berkeley, Los Angeles et Londres, University of California Press, 1998, p. 71-163.

14. Al-Cha 'rānī, 'Abd al-Wahāb, لواقح الأنوار في طبقات الأخيار [Lumières sur les hiérarchies des vertueux], Le Caire, Al-Mața ' a Al- 'Āmira Al-Sharqiyya, 1897.

15. Le mois qui précède Ramadan dans le calendrier musulman. La période qui suit le 20 marque l'approche du mois de Ramadan. L'auteur de ces vers est resté anonyme. Les mêmes vers ont été attribués à plusieurs poètes, dont le plus célèbre (et le plus probable) est Abu Nuwās. 
Le cheikh, scandalisé, quitte Bagdad pour La Mecque et reste là jusqu'à sa mort ${ }^{16}$.

Pour Cha 'rānī, et de fil en aiguille pour Chaghmoum, le cheikh en question a mal compris le sens des vers, car la référence à l'acte de boire dans de grands gobelets est une métaphore : les vers encouragent les musulmans à multiplier les actes de dévotion avant qu'il ne soit trop tard (c'està-dire avant la mort), et dans un contexte soufi ils encouragent le disciple ou le chercheur à multiplier les actes de sacrifice et d'ascétisme. Il s'agit donc d'une discipline spirituelle plutôt qu'une indulgence corporelle. Le malentendu est facile à comprendre : puisque les vers sont récités par un ivrogne, le cheikh suppose qu'il encourage ses auditeurs à boire beaucoup de vin, et partant, il fuit Bagdad, la cité corrompue, pour le lieu saint qu'est La Mecque. Al-Cha 'rānī en tire une leçon importante : seul celui qui souffre de cécité intellectuelle et spirituelle appellerait à la censure de la poésie et en empêcherait la transmission. Mis à part le rapport très évident entre ce roman et l'intérêt de Chaghmoum pour le soufisme, nous retenons deux éléments capitaux pour la lecture de Masālik Al-Zaytoun :

- Les aléas de la signification : les aspects figuratifs de la langue sont tels qu'une phrase n'est souvent compréhensible que par une interprétation qui contredit le sens apparent, d'où l'importance de la largeur d'esprit (intellectuellement parlant) du lecteur qui aborde le roman de Chaghmoum. Il s'ensuit que la même règle s'applique à l'être et au paraître : le premier contredit souvent le dernier, et les soufis, qui pourraient donner l'impression de contredire la loi de l'islam, ne font que l'appliquer, l'approfondir et la pratiquer.

- Le fait que le cheikh quitte Bagdad pour La Mecque implique que le chaos et le désordre moral de Bagdad sont neutralisés par la ville sainte de l'Islam.

Le lien entre la ville et le désordre mérite d'être lu plus profondément. La ville, comme nous le rappelle Richard Lehan, est l'endroit où l'homme se sépare de la nature, mais cette séparation n'est pas sans tension : c'est la ville qui abrite et protège l'homme contre les forces de la nature, mais qui se voit comme assiégée par d'autres forces qu'elle génère elle-même. Le locus classicus de cette histoire - de la figure de l'autre qui incarne les forces mystérieuses qui troublent l'ordre urbain - est la tragédie d'Euripide, Les Bacchantes, où Dionysos revient à Thèbes sous la forme d'un étranger pour s'emparer de sa ville natale qui, entretemps, a cessé de l'honorer. La cruauté de Dionysos, le dieu étranger, est telle qu'il fait en sorte qu'Agave, la mère de Penthée, roi de Thèbes, finit par tuer son fils (voire le déchirer), aidée par les autres bacchantes. L'altérité de Dionysos se reflète dans l'aliénation des bacchantes, l'autodestruction de l'ordre de la ville vient de l'intérieur de ses 
habitants, à commencer par le roi et sa mère ${ }^{17}$. L'illusion qui rend cet acte possible est celle promulguée par Dionysos, qui fait croire à Agave que son fils n'est qu'un lion. Il incombe à Cadmos, le père d'Agave, de démontrer à sa fille la vérité de ce qu'elle a fait.

Dans Masālik Al-Zaytoun, nous avons deux références très claires à Dionysos ${ }^{18}$. La première arrive très tôt dans le roman. Un groupe d'amis est assis dans un bar où ils discutent des sujets associés à ce dieu : l'amour sexuel, le vin, la métamorphose. Dionysos, le dieu des masques et de l'altérité, fait sentir sa présence par la technique narrative : les personnages n'ont pas de nom, ils sont désignés par des lettres. La première page du roman raconte la légende platonique de l'amour, selon laquelle l'homme n'aime dans la femme que la divinité qu'il y trouve, ce qui pousse le personnage G. à dire : «Bacchus est mon seigneur ${ }^{19}$. » À l'autre bout du texte, dans la deuxième partie du roman, nous trouvons une autre bacchanale qui tourne mal. Guidé par un cheikh, le narrateur décrit une suite de méditations et de visions extatiques. Le lecteur suppose qu'il est en train de suivre une initiation à une confrérie mystique. Dans le dernier chapitre, pourtant, le lecteur apprend que le narrateur n'a fait que subir une aliénation comparable à celle d'Agavé et des bacchantes : il a tué Zakiyya, la femme qu'il aimait, et ce qu'il voyait dans les rêves et visions de son initiation n'était que divers aspects de son corps et de son caractère. Dans un de ces rêves il se voit sur un nuage qui ressemble à une jument, et la description du corps de la jument revient sous la forme du corps de Zakiyya dans le dernier chapitre ${ }^{20}$. Même le cheikh qui devait le prendre en main, l'instruire et l'initier s'avère être le policier qui mène l'enquête. Il va de soi que le lecteur doit interpréter cette scène « à l'inverse » selon la logique de l'anecdote tirée d'Al-Cha 'rānī : on comprend que, loin d'avoir été persuadé doucement à reconnaître les faits, le narrateur a sans doute été torturé.

Entre ces deux parenthèses dionysiaques, le déroulement du roman oscille entre les corps des divers personnages et la ville de Meknès, tout comme le corps de Zakiyya se métamorphose en nuage et le nuage en jument. Ayant suggéré le sens dionysiaque et mystique du roman via

17. Cet aspect du mode d'opération de Dionysos est analysé par Albert Henrichs dans son article, " "He Has a God in Him": Human and Divine in Modern Perceptions of Dionysus », dans T. H. Carpenter et C. A. Faraone (dir.), Masks of Dionysus, Ithaca et Londres, Cornell University Press, 1993, p. 13-43.

18. Elles ne sont pas les seules, quoique les plus claires. On trouve d'autres allusions à Dionysos dans l'œuvre de Chaghmoum, notamment dans Ariāna (Ariane est devenue l'amante de Dionysos après avoir été abandonnée par Thésée). La dernière ligne du roman réunit les thèmes chers à Dionysos : " Je danse, comme je prie, ou je meurs. » (Miloudi Chaghmoum, Euvres complètes, op. cit., t. III, p. 266.)

19. Ibid., t. I, p. 447.

20. Ibid., p. 547-548 et p. 574-575. Ce passage marque un autre lien au sacré à travers l'allusion au voyage nocturne (isra $\left.{ }^{\prime}\right)$ et l'ascension céleste ( $\left.m i^{\prime} r \bar{a} j\right)$ du prophète Muhammad. 
l'épigraphe et la scène du bar dans le premier chapitre, le romancier perçoit Meknès à travers la forme féminine : « Je jure par Dieu que si tu voyais les cèdres dans ses yeux, les raisins dans ses joues, la menthe dans ses lèvres, tu ne pleurerais pas l'Andalous ni ton lieu de naissance. » À la page suivante, le narrateur rencontre une jeune femme d'une grande beauté, qu'il essaie de séduire. C'est une étudiante en arts qui attend le début d'un cours de sculpture. Il lui propose de l'appeler « raisin », mais elle refuse et insiste pour être appelée « Zaytoun» (olive), et puis, lorsqu'elle met ses mains sur ses joues, il en sort une espèce d'huile verdâtre ${ }^{21}$. Cette femme est donc la personnification de la ville de Meknès. Puis le narrateur lui demande sa robe, pour écrire un texte sur celle-ci. Le texte porte le même titre que le roman, Masālik Al-Zaytoun ${ }^{22}$. Cette identification entre la femme et la ville continue pendant la longue méditation sur l'histoire de Meknès qui suit ce chapitre :

Nos femmes ont été perdues par les conquérants, mutilées dès l'âge des Phéniciens jusqu'à celui des corsaires de l'océan du pétrole [les Arabes du Golfe]... (Je parle de la ville non de la femme)... Meknès n'est pas une ville comme les autres, Meknès est une ville transformée en village, plusieurs villes ont subi cette transformation et sont perdues, sauf Meknès qui se tient toujours debout et rêve d'être une capitale comme une vieille femme rêve d'être une princesse inégalée parmi les femmes et la seule désirée par les hommes ${ }^{23}$.

Cette personnification de la ville est en communication directe avec le corps du narrateur qui change de forme en fonction de la ville et de la femme qu'il voulait séduire et qui l'a séduit à son tour. Harcelé puis laissé tranquille par les forces de l'ordre dès qu'il commence à écrire, le narrateur termine le chapitre par un autoportrait qui souligne son rapport à l'univers physique : de même que Meknès est irriguée et pleine d'eau, lui aussi commence à gonfler ${ }^{24}$. Le transfert d'identité entre la belle fille, la ville de Meknès et le corps du narrateur est accompli ${ }^{25}$. Nous ne sommes pas loin des privilèges de l'artiste décrits par Baudelaire dans Le Spleen de Paris : « Le poète jouit de cet incomparable privilège, qu'il peut à sa guise être lui-même et autrui. Comme ces âmes errantes qui cherchent un corps, il entre quand il veut dans le personnage de chacun ${ }^{26}$. »

21. Miloudi Chaghmoum, Euvres complètes, op. cit., t. I, p. 467.

22. Ibid., p. 469.

23. Ibid., p. 474.

24. Ibid., p. 472.

25. D'autres interprétations du gonflement du narrateur sont possibles : selon Al-Alaoui, c'est une allusion à Al-Ḥallāj, qui selon la légende a gonflé jusqu'à ce qu'il lui soit devenu impossible de sortir de chez lui. On pourrait y repérer aussi une allusion à une des épithètes de Dionysos : phleus, ou le dieu gonflé. Voir Hicham Al-Alaoui, op. cit., p. 108.

26. Charles Baudelaire, Euvres complètes, éd. Claude Pichois, Paris, Gallimard, 1975-1976, t. I, p. 291. 
Désormais le corps, voire le moi, du narrateur est Meknès. Lorsque le narrateur révèle son passé dans un chapitre raconté à la deuxième personne, le lecteur comprend qu'il s'agit d'un homme qui a quitté sa ville natale dans le Sud, située non loin de Casablanca (tout comme Ben Ahmed, la ville natale de Chaghmoum) suite à certains traumatismes familiaux (mort du père; déchéance morale de la sœur; banqueroute de l'employeur; échec d'une carrière). Il arrive à Meknès, fait une dépression nerveuse et finit par tuer la femme qu'il aime et chez qui il loge. La description de son état mental et affectif se fait entièrement à travers l'usage du sens symbolique des murs de la ville, qui protègent et séparent en même temps, expression de son isolement et de sa frustration. En effet, Meknès n'est qu'un « labyrinthe de murs » dans lequel le narrateur se perd et dont il n'arrive pas à se sauver ${ }^{27}$. L'adéquation entre l'intérieur et l'extérieur continue sans trêve : «Je regarde ces murs jour et nuit [...] quand je les regarde c'est la tristesse et non l'orgueil qui me remplit [...] quarante kilomètres de murs sur la terre, combien y en aurait-il dans les ventres ${ }^{28}$ ? » Tant et si bien que les murs se trouvent « devant nous, dans nos lits, dans notre nourriture et nos vêtements $[\ldots]$ les murs possèdent des yeux et des mains ${ }^{29}$ !» Et le romancier se demande, « comment se libérer de la prison des oliviers [asr al-zaytoun $]^{30}$ ? ».

Toujours est-il que ce miroitement entre le narrateur et la ville n'est pas innocent. Suivant l'argument de Pavel, le roman dépend d'une séparation, une coupure entre le moi et le monde. Le rapport amoureux (à sens unique) qui lie le narrateur à Zakiyya, une aimable collègue qui l'aide et chez qui il loge, mais qui se prostitue, en dit long sur la situation problématique de ce narrateur : sa capacité à habiter le monde est liée directement à une femme qui vend son corps, qu'il aime et qui ne lui appartient pas. Il en va de même pour la prostituée R qui fréquente le même bar que le narrateur, qui l'invite chez elle à plusieurs reprises et qui se voit refusée. La dimension considérable de ce problème pousse le récit jusqu'à sa fin, dans une très longue suite de méditations et de fantasmes dans la deuxième partie, où le narrateur vole dans l'air, nage sous l'eau, entrevoit le corps de Zakiyya sans l'identifier avant de comprendre qu'il l'a tuée. La révélation est annoncée par le « cheikh » qui n'en est pas un, aidé par deux autres officiers qui se changent en rats. La bacchanale du début se reflète dans la farce de la fin du roman.

Ayant vogué un peu partout dans la ville de Meknès d'une manière qui rappelle Joyce, ayant subi bon nombre de métamorphoses - le narrateur « devient» la ville et plusieurs êtres dans la ville, dont le plus important

27. Miloudi Chaghmoum, Euvres complètes, op. cit., t. I, p. 478.

28. Ibid., p. 478-479. Le mot al-arhām suggère une multiplication biologique effrénée de la ville.

29. Ibid., p. 480.

30. Ibid. 
est les chiens de la ville qui personnifient son cynisme ${ }^{31}$ - le narrateur finit en meurtrier inconscient de ses actes. Cette fin marque un moment de crise dans la ville, tout comme dans Les Bacchantes d'Euripide. Si cette tragédie raconte la résistance acharnée au nouveau et à la transformation (et à l'impossibilité de survivre à cette résistance), le roman de Chaghmoum en dit long sur les versions contemporaines de cette crise, sur les vagues successives d'ordre et de désordre qui surgissent un peu partout dans la ville et qui affligent le narrateur. Bien que leurs origines soient étrangères, elles ont une qualité indigène (tout comme Dionysos qui naît à Thèbes mais qui se déguise en étranger). Elles sont donc impossibles à résoudre et restent incompréhensibles : la ville est à tout jamais dangereuse, agressive. Elle ne peut plus contenir les formes de violence qui naissent dans ses murs, parce qu'elle ne remplit plus sa fonction primaire, à savoir la protection. L'espace sacré de la ville qui devrait théoriquement protéger les citadins des forces qu'ils ne comprennent pas et qui les dépassent est effectivement désacralisé. Les institutions de la ville ne marchent pas : la langue n'est pas fiable et les personnages ne sont pas identifiables. Même celui qui est censé représenter l'autorité morale de la ville, le cheikh, n'est qu'un policier tortionnaire, et la pratique qui devait permettre au narrateur d'accéder à un ordre supérieur de vérité selon la voie soufie, ne fait que masquer un acte de violence dirigé contre celle qui l'a aidé. Langue, identité et éthique dérapent. Là où un certain diagnostic aurait été possible dans la tragédie d'Euripide - le roi impie est puni pour avoir déshonoré le dieu de la ville de Thèbes - ici rien de tel ne peut advenir. La ville n'est donc pas cette frontière entre l'homme et la nature; elle est en fait minée par la nature, autant par les éléments que par la psyché des citadins. Cette subversion des attentes de la ville, cette déconstruction de ce que la ville est censée faire, démontre la valeur du roman de Chaghmoum dans la mesure où la ville n'est plus le lieu de la culture ni de la civilisation, mais plutôt celui où l'ordre humain prend fin pour céder la place à quelque chose de plus sombre et de plus effrayant.

\author{
Ziad Elmarsafy \\ University of York (Royaume-Uni) \\ Department of English and related literature
}

31. Cet aspect du roman est un topos dans les romans de Chaghmoum : on voit la même identification entre le cynique et le chien dans Jazìrat al- 'ayn son premier roman (Ibid., p. 7). 